Gentlemen who have had one or two unfortunate results from its use should try it again. I am sure they will be pleased with it if properly used, in the form of a powder, in selected cases. In some cases it is with me the last resort, in others it is the first.

\section{THE TREATMENT OF THE MALPOSITION OF THE LID BORDER IN TRACHOMA.}

Presented to the Section on Ophthalmology at the Forty-eighth Anuual Meeting of the American Medical Association, at Philadelphia, Pa., June, 1-4, 1897.

BY F. C. HOTZ, M.D.

CHICAGO, ILL.

It is needless to say that the faulty position of the lid border induced by trachoma and known as entropium, can be treated only by operations. And it is also unnecessary to state that there is scarcely any other malformation of the human body for which a greater number of operations has been invented. Yet it is hardly reasonable to suppose that a condition so typical as the trachoma entropium could be treated with equal success by methods based upon widely different principles.

It is certainly very desirable that some order be brought into this chaos, and I believe this task is not so difficult as it may appear, if we clearly formulate our demands of what, in our judgment, a rational operation for entropium should and should not do.

The first condition we must insist upon, is that we should always tulie into serious consideration the cosmetic effect of our operation. The eyelids play a very important role in facial expressions, and we have no right to mar the face by unnecessarily mutilating the eyelids in the name of surgery. Every entropium operation which permanently disfigures the eyelid should be forever stricken from the list of legitimate ophthalmic operations; for the malposition of the lid border can well be rectified without the least mutilation.

The second condition we must insist upon is that the most rational method of relieving the entropium is to remove those structural changes which cause the inversion.

It is a prevalent idea that the entropium is caused by the shrinkage of the tarsal cartilage and the cicatricial contraction of the palpebral conjunctiva; and many operations are based upon this view. But that these structural changes are not the real etiologic factors in the production of entropium is evidenced by the fact that in many eyelids we find extensive cicatricial shrinkage without entropium, while in other lids we find its margin completely inverted though its tarsus is not only not contracted but abnormally large. Furthermore, it is well known that the destruction of the palpebral conjunctiva by burns does not lead to entropium, and that we may excise large portions of the palpebral conjunctiva and the cartilage without fear of producing entropium.

To trace the etiology of entropium we must study the affection in its incipient stage. And if we do so, we notice the very significant fact that the structural changes begin at the anterior edge of the lid margin. Long before the posterior edge shows the slightest disturbance, and even before the position of the lid margin itself is perceptibly altered, do the eyelashes show a decided drooping and the skin along their roots forms a fold falling down over and hiding the anterior edge of the lid border. In other words the skin and the cilia have slipped down on the outside of the tarsus.

This dislocation of the skin, which is shared by the lower bundles of the orbicularis muscle, is undoubtedly brought about by continued and oft-repeated spasms of the orbicularis muscle; for it is exactly the condition of the lid margin during acute blepharospasms, made permanent.

These anatomic changes at the anterior edge persist through all stages of entropium from beginning to end and are, I believe, the chief cause of the subsequent inclination of the lid border itself; for the displaced bundles of the orbicularis muscle resting upon the anterior edge of the lid margin exert a strong pressure upon the same by their contraction in the act of winking; the lid margin will gradually yield to this pressure, become stretched and inclined when its tissues are rendered less firm and resistent by the inflammation which usually invades the tarsus in the course of trachomatous conjunctivitis.

The foregoing exposition of the primary structural changes leading to the malposition of the lid border in trachoma plainly indicates what the principal aim of our treatment should be. The dislocated skin and muscle must be drawn up upon the external surface of the tarsus and fastened to it to prevent their slipping down again; the skin thus drawn up is to be stretched sufficiently so as to turn up by its traction the drooping cilia and the inclined lid margin.

These problems are solved by the following opertation: The lid skin being well put on a stretch, a transverse incision extending from canthus to canthus is made through skin and muscle a little below the upper border of the tarsus. The lid portion of the skin and muscle thus divided from the supratarsal portion is dissected up from the tarsus down to the roots of the eyelashes, and the muscular fibers covering the upper border of the tarsus are excised. The lid skin is then drawn up over the tarsus and fixed to its upper border by three silk or catgut sutures, which enclose within their loops only the skin borders of the incision and the upper tarsal border and, therefore, when tied, close the original wound and establish a firm union of the skin with the tarsus. In the five minutes allowed for this paper it is impossible to give a detailed description of the technique of this operation; nor is it necessary since I have fully described it on several former occasions.

Professor Schnabel ${ }^{2}$ of Prague, has modified the operation by making the incision only 1 or 2 millimeters above the eyelashes, and after dissecting up the skin and orbicularis muscle to near the upper border of the tarsus, he slides the narrow strip of skin upward until the cilia and lid margin are turned up, and fixes it to the tarsus by a running suture. $\mathrm{H}_{\theta}$ wrote me that the reposition of the cilia and lid margin is always permanently achieved by this procedure, and that even in the worst forms of entropium he has no need of grooving or splitting the cartilage.

My observations on this point have led to a different result. After I had introduced the operation at the Illinois Eye and Ear Infirmary, I noticed some of my colleagues invariably made the incision as low as Professor Schnabel; but the results were so much less satisfactory than my own, that in my second paper ${ }^{3}$ I took occasion to warn against placing the

1 Prager Med. Woch., 1893.

Archives of Ophthalmology, Vols. vili and xi; Annals of Ophthalmol. Vol. $v$. 
incision too near the free edge. But in many cases the cartilage is so small that the incision has necessarily to be made very close to the cilia. And I frankly admit that under these circumstances $I$ am not able to accomplish the complete and permanent reposition of the cilia by the above operation alone; $I$ find it necossary to add to it the intermarginal incis. ion and to fill the marginal wound with a graft of skin or mucous membrane. The graft should be of the same length and width as the wound. Retaining sutures are not necessary, but both eyes should be bandaged until the graft is well adherent in its new bed (twenty-four to forty-eight hours). I use skin grafts in preference to mucous membrane, because the normal lid margin is covered with skin, not with mucous membrane; because skin grafts are less liable to drying up and mortifying and because they give a more substantial support to the cilia. I cut the required strip from the integument behind the ear, the incisions penetrating obliquely just to the corium The graft is transported directly to the lid and adjusted to the wound.

Hairs do not grow in such skin grafts. If afterward any hairs appear in the new lid margin, it is easy to prove they are cilia left behind in the posterior portion of the lid margin by a faulty execution of the intermarginal incision.

The management of entropium briefly outlined in this paper is the result of careful and critical observations and trials carried on during the past twenty years; and I feel warranted by a large clinical experience, in stating that the operative procedures here recommended satisfy every requirement of a rational operation. They do not cause any disfiguration of the lids; they rectify the primary structural changes causing the entropium; and they relieve the malposition perfectly and permanently.

\section{HINTS ON THE HYGIENE OF TRACHOMA AND THE INFLUENCE OF ALTITUDE AND CLIMATE.}

Presented to the Section on Ophthalmology at the Forty-eighth Annual Meeting of the American Medical Association at Philadelphia, Pa., June 1-4, 1897.

BY SWAN M. BURnetT, M.D., Рн.D. WASHINGTON, D. C.

To establish efficient rules for the hygienic management of a disease it is first necessary to have definite ideas in regard to its causation, the condition of the subject and the environment most conducive to its development.

In respect to trachoma our knowledge is sadly deficient as to the majority of these factors. We have not yet arrived at the unanimous acceptance of a microbic origin of the disease. While the probabilities, in view of recent bacteriologic studies, point to the existence of a specific micro-organism as concerned in some way in the production of trachoma, the most diligent researches have failed to find one which any considerable number of investigators have agreed upon as the definite trachomatous microbe. By others it is held that the disease is essentially one of the adenoid tissue of the conjunctiva, which an inflammation of that membrane brought about by any means whatever is capable of calling up in a person predisposed to it, and who is in a condition favorable for its outbreak. In other words, a positive and well established operating course for trachoma has not yet been agreed upon. Even its contagiousness per se, which was formerly accepted as one of the characteristics of the disease, is now questioned by many. It is acknowledged that the discharge from a trachomatous eye may contain micro-organisms which, when transported to another as yet unaffected conjunctiva, will set up an inflammation there, but this inflammation is not necessarily trachomatous. Any inflammation, however, is capable of setting in motion a train of processes which will end in an outbreak of trachoma if a tendency to the disease be present, just as an attack of pneumonia will hasten the appearance of tuberculosis in one predisposed to it. This matter of predisposition as applied to trachoma is one which has received considerable attention only recently. In former times it was considered a purely contagious affection to which all individuals were liable in the same degree, and this idea is still held by some.

The facts that have been elicited from a systematic investigation of the prevalence of trachoma among different people show, however, such a disparity of susceptibility among them that we are forced to consider race as an important factor in the establishment of a predisposition to the disease. Some races, as the negro in the United States, are practically immune even under the most unfavorable hygienic surroundings, while others, as the Irish, Polish Jews, Italians, etc., are especially liable and suffer greatly when there are favorable conditions for the development of the disease. With the acceptance of the theory of a dyscrasia or predisposition as probably the essential character of the affection, our ideas in respect to its hygienic management must differ somewhat from those based on the theory of its purely contagious nature. As, however, the idea of a dyscrasia has been considered seriously only within a very recent period, our knowledge of the nature of this predisposition is as yet very limited. This is a field which at present offers the most promising results for study and investigation. It must be borne in mind that here, as in all diseases of a demonstrated or supposed microbic origin, even with the acceptance of a dyscrasia or predisposition, we have to consider not only the microorganism but also the soil in which it grows and develops. We must have soil as well as seed. A large number of pathologic micro-organisms we have always with us everywhere, and in the conjunctival sac of perfectly healthy eyes there are often to be found organisms which, if they were sufficiently numerous and the condition for their growth favorable, would in a short time set up a morbid process; all they need is a proper condition of the soil. Hitherto we have been directing our attention exclusively to the microbe, its form, habits, modes of development, etc., to the almost utter neglect of the soil on what it grows and pabulum on which it feeds. It is evident, therefore, that any system of hygienic management which aims at anything like scientific exactness and sutisfactory practical results, must embrace a study of the condition of the soil on which the micro-organism grows as well as the size and shape of the organism itself. This general proposition applies to trachoma so far as it may be demonstrated to be a microbic disease. But if it shall be found that there is no specific microbe but that it is simply a disease of the adenoid tissue of the conjunctiva, there must still be something which starts the morbid process in motion, and the conditions for its action must be propitious. It seems probable that this beginning may be an inflammation of the 